\title{
Cognitive Efficacy of Quetiapine in Early-Onset First-Episode Psychosis: A 12-Week Open Label Trial
}

\author{
Sébastien Urben · Pierre Baumann • Sandra Barcellona • \\ Muriel Hafil • Ulrich Preuss • Claire Peter-Favre • Stéphanie Clarke • \\ Olivier Halfon $\cdot$ Laurent Holzer
}

Published online: 19 November 2011

(C) Springer Science+Business Media, LLC 2011

\begin{abstract}
Twenty-three adolescents with psychotic disorders, aged from 13 to 18 years, participated in a 12-week open label trial (17 adolescents completed the study) in order to examine the impact of quetiapine on clinical status and cognitive functions (encompassing processing speed, attention, short-term memory, long-term memory and executive function). An improvement in Clinical Global Impression and Positive and Negative Symptom Scale $(P$ 's $\leq 0.001)$ was observed. In addition, after controlling for amelioration of symptoms, a significant improvement was observed on one executive function $(P=0.044$; Trail Making Part B). The remaining cognitive abilities showed stability. In addition, we observed an interaction between quetiapine doses $(>300 \mathrm{mg} /$ day or $<300 \mathrm{mg} /$ day $)$ and time, where lower doses showed more improvement in verbal short-term memory $(P=0.048)$, inhibition abilities $(P=0.038)$ and positive symptoms $(P=0.020)$. The neuropsychological functioning of adolescents with psychotic disorders remained mainly
\end{abstract}

\footnotetext{
S. Urben $(\bowtie)$

Unité de Recherche, Service Universitaire de Psychiatrie de l'Enfant et de l'Adolescent (SUPEA), Centre Hospitalier Universitaire Vaudois (CHUV), Route du Bugnon 25A,

1011 Lausanne, Switzerland

e-mail: Sebastien.Urben@chuv.ch

P. Baumann

Département de Psychiatrie, CHUV, Prilly-Lausanne, Switzerland

S. Barcellona · M. Hafil · L. Holzer

Centre Thérapeutique de Jour pour Adolescents, SUPEA, CHUV, Lausanne, Switzerland

U. Preuss

Kinder und Jugendpsychiatrische Poliklinik, Bern, Switzerland

U. Preuss

Klinik für Kinder- und Jugendpsychiatrie, Brandenburg, Germany

C. Peter-Favre $\cdot$ S. Clarke

Division de Neuropsychologie, CHUV, Lausanne, Switzerland

O. Halfon

SUPEA, CHUV, Lausanne, Switzerland
} 
stable after 12 weeks of treatment with quetiapine. However, lower doses seemed to have a better impact on two components of cognition (inhibition abilities and verbal short-term memory) and on positive symptoms.

Keywords Schizophrenia - Psychosis · Adolescence - Cognition · Neuropsychology · Quetiapine · Antipsychotic treatment $\cdot$ Cognitive enhancer

\section{Introduction}

Psychotic disorders comprise a wide spectrum of disabilities ranging from positive (hallucinations, delusions) and negative (alogia, anhedonia, avolition, flat affect) symptoms [1] to cognitive impairments in attention, processing speed, working memory, long-term verbal memory and executive functions [2-6]. These cognitive deficits are related to poorer functional outcome [7, 8]. More specifically in adolescents with psychotic disorders, these cognitive disabilities interfered with a period of important educational, social and emotional development [9]. Therefore adolescents suffering from psychotic disorders are a major concern for clinicians or mental health institutions having to find the most optimal treatments alleviating symptoms as well as being well adapted and self satisfactory for this age group.

Atypical antipsychotics (e.g. olanzapine, risperidone, quetiapine) have shown good tolerability and proven to be effective in treating negative and positive symptoms as well as promising improvement on cognitive impairment in adults with schizophrenia [10-14]. In adolescents with psychotic disorders, the efficacy of atypical antipsychotics on positive and negative symptoms has also been proven in different studies [15-19]. More specifically, in this population, quetiapine has been shown to reduce symptoms, to be well tolerated and to be associated with low weight gain and low extra-pyramidal symptoms occurrence [17, 20-22]. Atypical antipsychotics share some pharmacological properties in that almost all of them are antagonists at dopaminergic $\mathrm{D}_{2^{-}}$and serotonergic $5-\mathrm{HT}_{2 \mathrm{a}}$ receptors, in relationship with their effects on positive symptoms and negative symptoms, respectively [23]. Quetiapine shares structurally some common properties with the antipsychotic drug loxapine, which is metabolized by N-demethylation to the antidepressant drug amoxapine [24]. Quetiapine is one of the atypical antipsychotic drugs also introduced for the treatment of bipolar depression and as that, it is considered to be a first line option in international guidelines [25]. Interestingly, quetiapine similarly to loxapine is also demethylated: its metabolite norquetiapine [24] is a potent norepinephrine reuptake inhibitor and a partial 5- $\mathrm{HT}_{1 \mathrm{a}}$ agonist. Serotonin and noradrenaline are implicated in cognitive mechanisms [26, 27]. To the best of our knowledge, only one comparative, randomized, single-blind, 6-month study explored cognitive improvement related to treatment by quetiapine compared to olanzapine in an adolescent population [9]. Results revealed no amelioration in attention, working memory, learning and memory or executive functions. Therefore, further clinical trials are needed in order to replicate and confirm these results.

Thus, the present clinical study aimed at measuring the impact of quetiapine on cognitive functions and clinical status in adolescents with psychotic disorders. The impact of quetiapine on cognitive functions was assessed by neuropsychological tests before and after 12 weeks of quetiapine treatment. This investigation is part of a study on the clinical effectiveness and tolerability of quetiapine in adolescents with non-affective psychotic disorders [28]. 


\section{Methods}

\section{Study Design}

The comprehensive design of this study as it was presented in details elsewhere [28] is summarized as follows: this was a two-site (Lausanne and Bern university hospitals) openlabel trial in adolescents with psychotic disorders, in which assessments of cognitive impact and clinical efficacy of quetiapine were performed over 12 weeks. The study was approved by the Ethical Committee of the Lausanne University Hospital. Written informed consent was obtained prior to any study procedures by all patients and parents or legal representatives as required. Treatment discontinuation and study withdrawal could be motivated at any time by the subjects or the investigators.

\section{Inclusion Criteria}

Adolescents (13-18 years old) who fulfilled the DSM IV criteria for schizophrenia (295.10, 295.20, 295.30, 295.60, 295.90), schizophreniform disorder (295.40), schizoaffective disorder (295.70), delusional disorder (297.1), brief psychotic disorder (298.8), substance-induced psychotic disorder (293.xx), psychotic disorder NOS (298.8), mood disorders with psychotic features (296.x4) and who had no current medication or for whom initial antipsychotic treatment showed lack of efficacy or poor tolerance were targeted.

\section{Exclusion Criteria}

Subjects were excluded from the study if they showed (1) evidence of substance abuse (positive urine screening to opiates or ecstasy). Some cannabis consumption was not considered as exclusion criteria as this behavior is frequent in this population, for which effective treatments are needed; (2) a history or present condition of organic mental disorders, (3) mental retardation (IQ $<70$, defined as need for special education, assessed through a screening of the medical records about the activities before the first psychotic episode), (4) clinically meaningful non stable cardiovascular, (5) hepatic or renal diseases, (6) leucopenia $\left(\mathrm{WBC}<4,000 / \mathrm{mm}^{3}\right.$ ) or (7) pregnancy, or if they were currently enrolled in another medical trial.

\section{Patients}

A total of 23 participants were screened and selected in the study, 17 from center one (Lausanne) and 6 from center two (Berne). The adolescents were aged between 13 and 18 years (Mean \pm SD: $15.8 \pm 1.38$ years), 17 of them were male (6 females) and 20 were Caucasian. DSM-IV diagnoses included schizophrenia (7), schizophreniform disorder (6), schizoaffective disorder (1), psychotic disorder not otherwise specified (6), delusional disorder (2) and brief psychotic disorder (1). All 23 subjects received quetiapine, 17 completed the treatment (Completer) and 6 discontinued early (Non-completer) due to consent withdrawal (3), lack of compliance (1), lack of efficacy (1) and the occurrence of a serious adverse event (1).

Psychiatric comorbidities were present in the medical history of 11 patients: Eating disorder (1 patient), obsessive compulsive disorder (1), sexual abuse by an adult (1), substance abuse (1), suicide attempts (1), conduct disorder (2), depression and self-injuries (1), 
depression (1), onset psychosis as a child (1), social anxiety (1). 14 patients presented other than psychiatric problems in their medical history: Dermatological pathologies (5), musculoskeletal system (4), endocrinological system (3) (including 2 patients with hyperprolactinemia), peripheral nervous system (2), respiratory system (2), gastro-intestinal tract (1), cardiovascular system (1), blood (1) and allergy (1).

\section{Premedications and Concomitant Medications}

Concomitant medications were given only if considered necessary for the participants' safety or well-being. A failure of previous ongoing antipsychotic treatments lead to enrolment in the present study and so was discontinued. Eight participants received antipsychotic medication before the start of the quetiapine treatment: only olanzapine (3); only risperidone (3); olanzapine and risperidone (1); olanzapine, levomepromazine and zuclopenthixol (1). A wash-out period of at least $24 \mathrm{~h}$ was observed for any participant receiving prior antipsychotic treatments. In addition, for drug-naïve patients, a short period of treatment with the benzodiazepines clorazepate, alprazolam or diazepam could be conducted if needed.

Hepatic enzyme-inducing agents (e.g. carbamazepine, barbiturates, glucocorticoids) had to be avoided as concomitant medication. No other psychotropic medication was allowed except alprazolam, diazepam, clorazepate in case of severe agitation, chloral hydrate in case of invalidant insomnia, biperidene when appropriate.

\section{Medication Dosing}

Doses were adjusted by senior psychiatrists of both sites (L.H. and U.P.) in function of efficacy and tolerability in order to improve the compliance and to limit side effects (as sedation, for example). Quetiapine doses (computed by summing the total amount of administered doses divided by the number of days of exposure) ranged between 42 and $858 \mathrm{mg}$, the mean daily dose was $350 \pm 213 \mathrm{mg} /$ days. Mean exposure time to quetiapine was $77 \pm 28$ days, with a minimum of 9 and a maximum of 106 days of exposure. Plasma concentrations of quetiapine as assayed by a previously described method [28] were $213 \pm 277 \mathrm{ng} / \mathrm{ml}$ at week $12(n=14)$. As about half of the patients received doses lower than $300 \mathrm{mg} / \mathrm{day}$, in the final part of the analyses the adolescents were split in function of the received doses ( $>300 \mathrm{mg} /$ day or $<300 \mathrm{mg} /$ day) in order to explore for possible differences of the effect of quetiapine doses on cognition and clinical status. The mean doses for the "low doses group" ( $n=8$ ) were $136 \pm 82 \mathrm{mg} /$ day (range 42-273 mg/day) and of $498 \pm 130 \mathrm{mg} /$ day (range 339-858 mg/day) for the "high doses group" ( $n=9$ ). Plasma levels of quetiapine confirmed the repartition of the adolescents (at week 12 it was of $14 \pm 9 \mathrm{ng} / \mathrm{ml}$ and of $363 \pm 287 \mathrm{ng} / \mathrm{ml}$ for the lower and higher doses group, respectively) in that, as expected, plasma levels of quetiapine were lower in the low dose than in the high dose group.

\section{Assessments}

\section{Clinical Status}

Participants were assessed with the Positive and Negative Symptom Scale (PANSS), assessing positive and negative symptoms as well as general psychopathology in subjects 
with schizophrenia and related disorders [29]. Clinical efficacy was evaluated with the Clinical Global Impression-Severity of illness scale (CGI) [30]. Evaluations were conducted both at baseline and at study week 12 .

\section{Neurocognitive Test Battery}

A comprehensive test battery was administered by a trained neuropsychologist at baseline and after 12 weeks of treatment. The psychometric tests were chosen because they represent main functions which can be altered in psychotic disorder, i.e. processing speed, attentional abilities, memory abilities and executive function $[2,5,6]$.

Processing speed ability was assessed with the Trail Making Test-Part A [31] where digits ranging from 1 to 25 had to be connected with a continuous line. In addition, the part of the Stroop task, where images were to be named, was also used to assess processing speed abilities. The time to realize the tests were recorded.

Selective and Sustained Attention were assessed with the Continuous Performance Test (CPT) degraded version [32, 33]. In this test, stimuli were presented to the participant who had to detect (by pressing on a key response) the occurrence of a target stimulus during an 8 -min period. The scores were the hit rate (mean success rate), the false alarm rate (responding to interfering stimuli), $d^{\prime}$ (computed from the proportion of hits and false alarms) representing a measure of signal/noise discrimination (i.e. the ability to discriminate between target and non-target).

Short term memory ability was assessed with two tests. Firstly, the Audio Span Test [34] was administered. In this task, clusters of numbers were read by the examiner and had to be repeated by the participant. Secondly, in the Visuo-spatial Span Test [35] visuospatial sequences were performed by the examiner and repeated by the subject in the same order. The scores were made of the maximal cluster of numbers or the maximal visuospatial sequence correctly repeated.

Long term memory ability was assessed with two tests. Firstly, the Rey Auditory Verbal Learning Test [36] which consisted in a list of 15 words that the participant had to learn through 5 immediate recall. Then, a delayed recall was required. The score used for this task was the number of words correctly recalled in the delay recall. Secondly, the complex figure of Rey [37] was used. This task consisted in copying and recalling a complex geometrical figure. The score was made out of the correct recall of the elements of the geometrical figure.

Executive functions were assessed with three tests. First, in the Verbal Fluency Task [38] as many words as possible of one category (letter $\mathrm{M}$ or animal) had to be generated in a limited time $(60 \mathrm{~s})$. The score was made of the total number of correct words expressed in both part (with the letter M and animal). Secondly, the Trail Making Test-Part B [31] was administered. In this task, participants had to connect digits and numbers alternatively. The time to realize this task and errors were recorded. The interference condition of the Stroop task requires suppressing the automatic response of word learning in favor of the less automatic process of object naming. Speed of naming (time score) and the number of interference errors were recorded.

\section{Additional Measures}

Urine sample was asked for illicit drug detection at week 0. Semi-quantitative urine analyses included the following substances: cannabis, benzodiazepines, opiates, cocaine, methadone and amphetamines. In addition, the substance use and frequency before and 
during the study were assessed by the substance use questionnaire derived from the Adolescent Drug Abuse Diagnosis [39]. Thus, at baseline, one participant injected heroin, and eight patients drunk alcohol 1-5 times during the past month (usually 1-6 drinks per day). Eleven participants smoked every day tobacco during the past 6-48 months. In addition, 10 participants smoked regularly [several times per week $(n=5)$ or per day $(n=5)$ ] cannabis during the past 12-48 months. Two participants had to be considered as polydrug users, as they reported to drink alcohol and smoke cannabis and tobacco, as well as to intake amphetamines, tranquilizers, cocaine, hallucinogens and phencyclidine.

\section{Statistical Analysis}

Descriptive statistics were provided for dependant variables. Everywhere, if not otherwise mentioned, data were presented as means \pm SD. As the data suit a Gaussian distribution, parametric tests were used. First, we tested the effect of antipsychotic pre-medication using independent tests of Student on the evaluation at baseline and at the end of the study. Then dependent sample tests of Student assessed the changes in performance on neuropsychological tests. After testing for main effect of quetiapine on cognition, analyses of covariance (with the amelioration of the symptoms as covariate) were computed [40]. Then, correlation analyses were conducted between changes in clinical status and changes in cognitive abilities. After that, 2 (quetiapine doses: higher or lower than $300 \mathrm{mg} / \mathrm{day}$ ) $\times 2$ (time: Baseline vs. at week 12) analyses of variance (ANOVA) were conducted in order to observe possible interaction effects between time and quetiapine doses. Finally, differences between patients who did (completer) and did not complete (non-completer) the study were assessed with independent sample tests of Student at baseline.

\section{Results}

\section{Pre-Medication Effect}

The effect of previous antipsychotic medication was tested at baseline and at the end of the study. Results revealed no significant differences on clinical status, cognitive abilities or rate of non completers between the participants who take antipsychotic medication prior to the study versus the participants who did not receive any antipsychotic before the study.

\section{Impact of Quetiapine on the Clinical Status}

At the end of the study, significant reduction was observed on the three subscales of the PANSS (positive, $P<0.001$; negative, $P=0.004$; general psychopathology subscales, $P<0.001)$. In addition, the severity of the symptoms decreased during the 12 weeks treatment $(P<0.001)$, (Table 1$)$.

\section{Impact of Quetiapine on Cognition}

Changes on neuropsychological testing are displayed in Table 2. A statistically significant improvement in processing speed was found regarding the denomination part (picture naming) of the Stroop task $(P=0.002)$ and the Part A of the Trail Making Test task showed marginal differences $(P=0.065)$. However, after controlling for the amelioration 
Table 1 Descriptive scores for the symptomatology

\begin{tabular}{lcrrcrr}
\hline Scale & $N$ & Baseline & At week 12 & \multicolumn{3}{c}{ Changes from baseline to week 12 } \\
\cline { 5 - 7 } & & Mean (SD) & Mean (SD) & Mean (SD) & \multicolumn{1}{c}{$t$} & \multicolumn{1}{c}{$P$} \\
\hline CGI Severity & 20 & $4.90(0.64)$ & $3.35(0.99)$ & $1.55(1.00)$ & 6.94 & $<0.001$ \\
PANSS Positive & 20 & $22.60(5.74)$ & $16.85(6.85)$ & $5.75(5.25)$ & 4.90 & $<0.001$ \\
PANSS Negative & 20 & $26.20(5.99)$ & $21.25(5.43)$ & $4.95(5.72)$ & 3.87 & 0.001 \\
PANSS Global & 20 & $48.30(7.98)$ & $37.55(9.23)$ & $10.75(10.23)$ & 4.70 & $<0.001$ \\
\hline
\end{tabular}

Mean scores on Clinical Global Impression-Severity of illness scale (CGI Severity) and on the general psychopathology (PANSS Global), negative symptoms (PANSS Negative) and positive symptoms (PANSS Positive) subscales of the PANSS: at baseline, at week 12 and changes

Table 2 Mean raw scores on neuropsychological testing: baseline, at week 12 and changes

\begin{tabular}{|c|c|c|c|c|c|c|c|}
\hline \multirow{2}{*}{$\begin{array}{l}\text { Cognitive } \\
\text { domain }\end{array}$} & \multirow[t]{2}{*}{ Test } & \multirow[t]{2}{*}{$N$} & \multirow{2}{*}{$\begin{array}{l}\text { Baseline } \\
\text { Mean (SD) }\end{array}$} & \multirow{2}{*}{$\begin{array}{l}\text { At week } 12 \\
\text { Mean (SD) }\end{array}$} & \multicolumn{3}{|c|}{ Change from baseline to week 12} \\
\hline & & & & & Mean (SD) & $t$ & $P$ \\
\hline \multirow{2}{*}{$\begin{array}{l}\text { Processing } \\
\text { speed }\end{array}$} & TMT A & 17 & $51.35(15.31)$ & $46.35(13.89)$ & $-5.00(10.41)$ & 1.98 & 0.065 \\
\hline & Stroop image & 18 & $40.28(8.87)$ & $37.28(7.63)$ & $-3.00(3.58)$ & 3.55 & $0.002 * *$ \\
\hline \multirow[t]{3}{*}{ Attention } & Hit rate & 14 & $67.32(22.20)$ & $67.80(19.46)$ & $0.47(15.41)$ & -0.12 & 0.910 \\
\hline & FA & 14 & $12.24(13.02)$ & $11.43(12.96)$ & $-0.81(2.78)$ & 1.10 & 0.293 \\
\hline & $d^{\prime}$ & 14 & $1.95(1.21)$ & 1.99 (1.13) & $0.04(0.59)$ & -0.24 & 0.813 \\
\hline \multirow[t]{2}{*}{$\begin{array}{c}\text { Short term } \\
\text { memory }\end{array}$} & $\begin{array}{l}\text { Auditory } \\
\text { span }\end{array}$ & 17 & $5.88(0.92)$ & $5.88(0.1 .16)$ & $0.00(1.00)$ & 0.00 & 1.000 \\
\hline & $\begin{array}{l}\text { Visuo-spatial } \\
\text { span }\end{array}$ & 17 & $5.41(1.12)$ & $5.59(1.22)$ & $0.18(1.42)$ & -0.51 & 0.616 \\
\hline \multirow{2}{*}{$\begin{array}{l}\text { Long term } \\
\text { memory }\end{array}$} & 15 Words & 17 & $11.24(3.01)$ & $10.35(3.79)$ & $-0.88(3.46)$ & 1.05 & 0.273 \\
\hline & Rey figure & 17 & $19.88(7.93)$ & $22.06(6.37)$ & $2.18(7.91)$ & -1.13 & 0.301 \\
\hline \multirow{3}{*}{$\begin{array}{l}\text { Executive } \\
\text { functions }\end{array}$} & Fluency & 17 & $32.53(5.62)$ & 33.12 (8.78) & $0.59(7.55)$ & -0.32 & 0.752 \\
\hline & $\begin{array}{l}\text { Stroop } \\
\text { inhibition }\end{array}$ & 18 & $59.17(22.81)$ & $51.94(10.07)$ & $-7.22(19.18)$ & 1.60 & 0.129 \\
\hline & TMT B & 17 & $81.41(27.53)$ & $68.35(26.86)$ & $-13.06(20.06)$ & 2.68 & $0.016 *$ \\
\hline
\end{tabular}

TMT Part A, Trail Making Test Part A, time in seconds; Stroop image, Stroop task, denomination of image part, time in seconds; Hit rate, CPT, mean success rate, percentage; FA, CPT, false alarm rate, percentage; d', CPT, sensitivity; auditory span, number of recalled numbers; visuospatial span, number of sequences correctly recalled; 15 words, Rey Auditory Verbal Test, number of words correctly recalled; Rey figure, complex figure of Rey, points; fluency; number of words expressed; Stroop inhibition, Stroop task, interference part, time in seconds; TMT B, Trail Making Test Part B, time in seconds. $* P<0.05 ; * * P<0.01$

of the symptoms (changes in CGI entered as covariate), this effect was only a trend regarding the denomination part of the Stroop task, $F(1,16)=3.54 ; P=0.078$; $\eta_{\mathrm{p}}^{2}=0.18$, and disappeared in the Part A of the Trail Making Test $F(1,15)=0.781$; $P=0.391 ; \eta_{\mathrm{p}}^{2}=0.00$. The Part B of the Trail Making Test showed significant differences between both administration $(P=0.016)$. This differences remained significant even after controlling for the amelioration of the severity of the symptoms (changes in CGI entered as covariate), $F(1,15)=4.82 ; P=0.044 ; \eta_{\mathrm{p}}^{2}=0.24$. Small changes that did not reach significance were found for the remaining tests. These differences were not influenced by substance abuse as well as by comedication as there were no significant differences in function of these variables, both at baseline and after 12 weeks. 


\section{Relation Between Cognition and Clinical Status}

Then, we conducted correlation analyses with Pearson's correlation in order to observe if the improvement in clinical status were related to improvement in the cognitive abilities. The analyses revealed no significant correlations between the improvement in symptomatology and cognition.

\section{Impact of the Doses on the Changes in Cognition and Clinical Status}

The quetiapine doses' groups did not differ in their number of male/female $\left(\chi_{(1)}^{2}=0.2\right.$, $P=0.658)$, substance abuse $\left(\chi_{(1)}^{2}=1.7, P=0.193\right)$ or concomitant medication $\left(\chi_{(1)}^{2}=\right.$ $2.2, P=0.138$ ). Thus, 2 (doses: higher or lower than $300 \mathrm{mg} /$ day) $\times 2$ (time: baseline vs. after 12 weeks) ANOVAs tested the effects of doses on the improvement of cognition and clinical status. The interaction effect was of particular interest in order to observe different influence of quetiapine doses on cognition and clinical status. The results revealed a significant interaction effect on the number of words recalled on the audio span task, $F(1,15)=4.64 ; P=0.048, \eta_{\mathrm{p}}^{2}=0.24$; on the time to realize the interference part of the Stroop task, $F(1,16)=5.14 ; P=0.038, \eta_{\mathrm{p}}^{2}=0.24$; and on the positive scale of the PANSS, $F(1,17)=6.53 ; P=0.020 ; \eta_{\mathrm{p}}^{2}=0.28$. Figure 1 illustrated these interactions. One could observe that lower doses showed more efficient improvement on verbal memory, inhibition and positive symptoms. However, $t$-test comparing the groups at baseline or after 12 weeks did not reveal significant differences.

\section{Completers Versus Noncompleters}

At baseline, no significant difference between the patients who did and did not complete the study was found regarding the PANSS and CGI. Regarding the cognitive measure, the completers differed from the non-completers in their performances in the fluency task $(P=0.005)$, non-completers giving less responses than completers.

\section{Discussion}

In this study, the clinical impact of quetiapine on positive and negative symptoms, as well as on general psychopathology (Table 1) confirms previous findings about positive effects of atypical antipsychotic medication $[18,19,41]$ and of quetiapine both in adults $[14,20$ $22]$ and in adolescents $[9,17]$. With regard to the effects of quetiapine on cognition, after 12 weeks of treatment, a significant improvement was observed in the executive function, more specifically in shifting abilities (Table 2), which remained significant after controlling for symptoms amelioration. This ability to shift between two or more tasks, operations or mental sets, refers to executive functions. Executive functions are important processes when using routines is not possible and to serve goal-directed behavior [42]. Thus, improvement in such abilities allows for better planning and problem-solving, for example. In contrast, processing speed seemed to improve, but this improvement was more related to the amelioration of the symptoms as the effect disappeared after controlling for this amelioration. Short-term memory, long-term memory and attention remained stable. These results were similar to those found in adults with psychosis, for whom significant executive functions improvements related to quetiapine's treatment have been found in shifting $[14,43]$ and inhibition abilities [44]. However, the present results contrast with those 

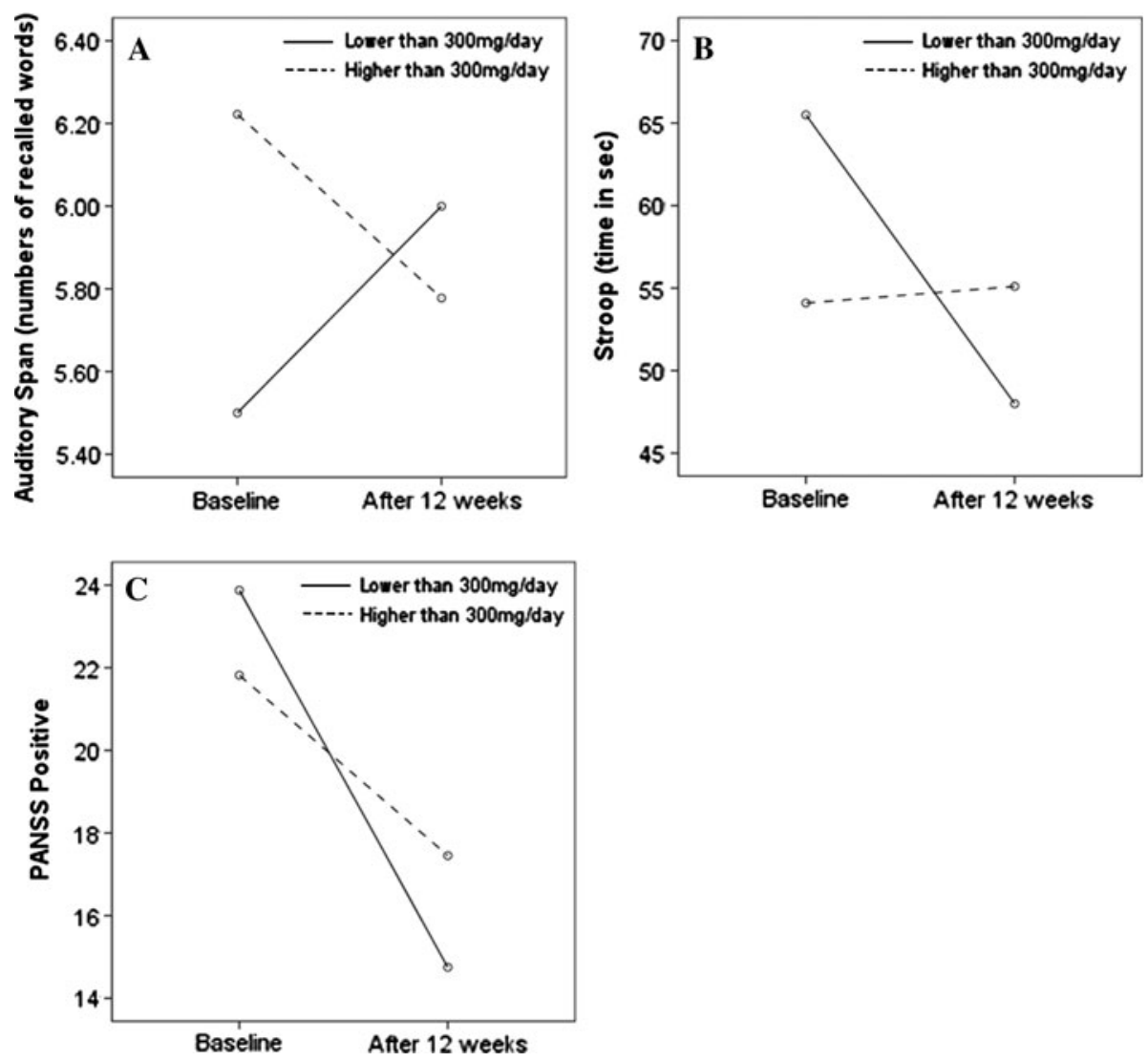

Fig. 1 Quetiapine doses by time interactions. Note Interaction effect between quetiapine doses and time for the auditory span (a), for the interference part of the Stroop task (b), and for the positive symptoms scale of the PANSS (c)

obtained by Robles et al. [9] comparing the effect of quetiapine and olanzapine on cognitive abilities of adolescents over 6 months who reported no amelioration of these abilities.

Treatment doses used in this study $(350 \pm 213 \mathrm{mg} /$ day quetiapine) were inferior to those usually administered in the adult population. However, a high variability in the administered quetiapine doses was also reported in previous trials with adolescent patients: $532.8 \pm 459.6 \mathrm{mg} /$ day (reported in $[9,17]$ ). Regarding the treatment's doses, inconsistent results were found in adult populations. Thus, Arvanitis and Miller [45] demonstrated that doses from $150 \mathrm{mg} /$ day were efficient in adult populations to reduce positive symptoms but that doses of $300 \mathrm{mg} /$ day at least are required to decrease negative symptoms. In contrast, Small et al. [46] demonstrated in adult populations that lower doses than $250 \mathrm{mg} /$ day might not be less efficient in order to improve positive symptoms. In adolescent populations the effective doses in order to improve clinical status seemed to vary between studies. Shaw et al. [21] administered $467 \mathrm{mg} /$ day (ranging from 300 to $800 \mathrm{mg}$ ) and Swadi et al. [22] reported a mean administered dose of $607 \mathrm{mg} /$ day and reported that 4 participants out of 11 had to receive higher doses than $1,000 \mathrm{mg}$ to be effective. However, in the present study, although the whole group showed improvement in clinical status, the adolescents who 
tolerated only lower doses seemed to show more improvement in positive symptoms than the patients receiving higher doses.

With regard to the cognitive abilities, the same interaction was found (Fig. 1) in inhibition and verbal short-term memory. Thus, participants receiving lower doses of quetiapine demonstrated more improvement in verbal short-term memory and inhibition of automatic response compared to adolescents with higher doses of quetiapine. Both cognitive abilities are important in everyday life. Thus, short-term retention of information helped to restrain information in many situations of life and resisting to automatic behavior in favor of a non dominant one allowed adopting more appropriate behavior. Contrasting results were found in adults with schizophrenia. Velligan et al. [11] suggested that $600 \mathrm{mg} /$ day doses of quetiapine allow a higher improvement in executive function and verbal memory than lower doses (300 mg/day). Nevertheless, to our knowledge, no study explored the efficiency of different doses in adolescent populations. Thus, quetiapine could have different impact according to the chronicity of the illness and the age of the patient. Indeed, some studies demonstrated that compared to those with chronic illness, treatment, even with lower doses (often 50\% lower than doses for chronic patients), of individuals in first-phases of psychosis tended to be more efficient in terms of symptoms reduction [47-49]. These effects could be explained by the changed sensitivity of $\mathrm{D}_{2}$ receptors due to long-term treatment [50]. Considering all above studies, further trials are warranted to determine the more effective doses of quetiapine in order to treat psychotic symptoms and improve cognitive abilities in adolescent populations.

The action of quetiapine in the brain could help us to understand this result. Quetiapine is associated with the occupancy of $\mathrm{D}_{2}$ (dopamine) and $5 \mathrm{HT}_{2 \mathrm{a}}$ (serotonin) receptors which mediates antipsychotic effect (for a recent review see [51]). In adult populations, data suggested that lower doses than $450 \mathrm{mg} /$ day did not allow an adequate occupancy of the $\mathrm{D}_{2}$ receptor [46, 52-54] and so would not be effective. However, no study explored the effect of quetiapine on brain of adolescents where the necessary occupancy of $\mathrm{D}_{2}$ and $5 \mathrm{HT}_{2 \mathrm{a}}$ receptors could be sufficient at lower doses of quetiapine or that the effective occupancy of receptor could be reached with lower doses. Further studies are needed to determine the efficient doses of quetiapine to have a sufficient occupancy of $\mathrm{D}_{2}$ receptor. These considerations, however, neglect the fact that, as presented in the Introduction, quetiapine also exerts by its metabolism to norquetiapine, agonistic serotonergic (as a partial 5-HT1a) and noradrenergic (as a noradrenaline reuptake inhibitor) effects [24, 51]. These neurotransmitters have an important impact on psychomotor and vigilance functions, respectively [52]. Due to the non-availability of norquetiapine for analytical purposes, it could not be analyzed in this study, but a previous investigation shows that in schizophrenic patients treated with quetiapine, norquetiapine is present in measurable concentrations both in plasma and CSF [55]. In addition, the treatment resulted in a 35 and 33\% increase in CSF 5-HIAA and MHPG, metabolites of serotonin and noradrenaline, respectively. Moreover, significant correlations were calculated between norquetiapine concentrations in CSF and the changes of 5-HIAA $(P<0.01)$ and MHPG $(P<0.03)$ CSF levels.

Limitations of the present study emanate from the study design. Despite the fact that the study was planned and executed by independent practitioners and data analysis was verified by an independent statistician, this was an open-label study, with no control group. There is no indication of spontaneous recovery. Moreover, associations between cognitive and clinical improvements are likely to appear at a general level, but are not necessarily found at the individual level [56]. In addition, as the group was divided a posteriori in function of the effectiveness and side effect of quetiapine treatment, further studies are 
needed, in order to explore in more details the effect of low doses of quetiapine on cognition and clinical status. Finally, practice effect may have interfered in the evaluation of the cognitive improvement. However, a study conducted on schizophrenic outpatient showed a very low rate of practice effect over 10 weeks on tests used in the present study such the TMT, HVLT or the forward and backward digit recall [57]. Consistently, a previous study reported a lack of test-retest effect in first-episode schizophrenic patients [14]. A further limitation of the study is the prior treatment by other antipsychotics for some patients $(N=8)$. However, those patients were enrolled because of a lack of efficacy of the treatment and therefore during the study the patients were treated only with quetiapine. Furthermore, our analyses revealed no differences at baseline or at the end of the study when we compared the patients who take previously an antipsychotic or those who did not take previously an antipsychotic medication.

In conclusion, as cognitive functions play a major role in predicting psycho-social functioning $[7,8]$ and as adolescence is a critical period of development in many aspects of life, it is important to develop effective therapeutic programs which target cognitive impairments. Given the greater cerebral plasticity in this age-group, approaches which combine antipsychotic medication and cognitive remediation may enhance treatment effects and may be worth investigating. However, further studies are needed to ensure that the core feature, i.e. cognitive dysfunctions, in adolescence psychosis can be targeted by medication and/or cognitive remediation, and that substantial improvement is followed by better functional outcome. Finally, by its unique pharmacological profile, the effects of quetiapine and especially of norquetiapine should be further examined, also in animal models of cognitive functions.

Acknowledgment This study was financially supported by AstraZeneca, Switzerland, by an unrestricted grant.

Conflict of interest PB and UP have received honoraries or sponsorships from almost all pharmaceutical companies in Switzerland selling psychotropic drugs. In particular, they received honoraries from the manufacturer of quetiapine (AstraZeneca). Others authors reported no conflicts of interest.

\section{References}

1. APA: Diagnostic and Statistical Manual of Mental Disorders (DSM-IV), 4th edn. Washington, DC, American Psychiatric Association, 1994

2. Asarnow RF, Asamen J, Granholm E, et al.: Cognitive/neuropsychological studies of children with a schizophrenic disorder. Schizophrenia Bulletin 20:647-669, 1994

3. Holzer L, Chinet L, Jaugey L, et al.: Detection of cognitive impairment with the Repeatable Battery for the Assessment of Neuropsychological Status (RBANS) in adolescents with psychotic symptomatology. Schizophrenia Research 95:48-53, 2007

4. Kenny JT, Friedman L, Findling RL, et al.: Cognitive impairment in adolescents with schizophrenia. American Journal of Psychiatry 154:1613-1615, 1997

5. Kumra S, Wiggs E, Bedwell J, et al.: Neuropsychological deficits in pediatric patients with childhoodonset schizophrenia and psychotic disorder not otherwise specified. Schizophrenia Research 42:135-144, 2000

6. Rhinewine JP, Lencz T, Thaden EP, et al.: Neurocognitive profile in adolescents with early-onset schizophrenia: Clinical correlates. Biological Psychiatry 58:705-712, 2005

7. Green MF: What are the functional consequences of neurocognitive deficits in schizophrenia? American Journal of Psychiatry 153:321-330, 1996

8. Hofer A, Baumgartner S, Bodner T, et al.: Patient outcomes in schizophrenia II: The impact of cognition. European Psychiatry 20:395-402, 2005 
9. Robles O, Zabala A, Bombin I, et al.: Cognitive Efficacy of quetiapine and olanzapine in early-onset first-episode psychosis. Schizophrenia Bulletin 37:405-415, 2011

10. Riedel M, Spellmann I, Strassnig M, et al.: Effects of risperidone and quetiapine on cognition in patients with schizophrenia and predominantly negative symptoms. European Archives of Psychiatry and Clinical Neuroscience 257:360-370, 2007

11. Velligan DI, Newcomer J, Pultz J, et al.: Does cognitive function improve with quetiapine in comparison to haloperidol? Schizophrenia Research 53:239-248, 2002

12. Zhong KX, Sweitzer DE, Hamer RM, et al.: Comparison of quetiapine and risperidone in the treatment of schizophrenia: A randomized, double-blind, flexible-dose, 8-week study. Journal of Clinical Psychiatry 67:1093-1103, 2006

13. Tandon R: Improvement without impairment: A review of clinical data for quetiapine in the treatment of schizophrenia. Journal of Clinical Psychopharmacology 23:S15-S20, 2003

14. Andersen R, Fagerlund B, Rasmussen H, et al.: Cognitive effects of six months treatment with quetiapine in antipsychotic-naïve first-episode schizophrenia. Psychiatry Research 187:49-54, 2011

15. Quintana H, Wilson MS 2nd, Purnell W, et al.: An open-label study of olanzapine in children and adolescents with schizophrenia. Journal of Psychiatric Practice 13:86-96, 2007

16. Sanford M, Keating GM: Aripiprazole: In adolescents with schizophrenia. Paediatric Drugs 9:419-423, 2007

17. Arango C, Robles O, Parellada M, et al.: Olanzapine compared to quetiapine in adolescents with a first psychotic episode. European Child and Adolescent Psychiatry 18:418-428, 2009

18. Findling RL, McNamara NK, Youngstrom EA, et al.: A prospective, open-label trial of olanzapine in adolescents with schizophrenia. Journal of the American Academy of Child and Adolescent Psychiatry 42:170-175, 2003

19. Dittmann RW, Meyer E, Freisleder FJ, et al.: Effectiveness and tolerability of olanzapine in the treatment of adolescents with schizophrenia and related psychotic disorders: Results from a large, prospective, open-label study. Journal of Child and Adolescent Psychopharmacology 18:54-69, 2008

20. McConville BJ, Arvanitis LA, Thyrum PT, et al.: Pharmacokinetics, tolerability, and clinical effectiveness of quetiapine fumarate: An open-label trial in adolescents with psychotic disorders. Journal of Clinical Psychiatry 61:252-260, 2000

21. Shaw JA, Lewis JE, Pascal S, et al.: A study of quetiapine: Efficacy and tolerability in psychotic adolescents. Journal of Child and Adolescent Psychopharmacology 11:415-424, 2001

22. Swadi HS, Craig BJ, Pirwani NZ, et al.: A trial of quetiapine compared with risperidone in the treatment of first onset psychosis among 15- to 18-year-old adolescents. International Clinical Psychopharmacology 25:1-6, 2010

23. Richelson E: New antipsychotic drugs: How do their receptor-binding profiles compare? Journal of Clinical Psychiatry 71:1243-1244, 2010

24. Jensen NH, Rodriguiz RM, Caron MG, et al.: $N$-Desalkylquetiapine, a potent norepinephrine reuptake inhibitor and partial 5-HT1A agonist, as a putative mediator of quetiapine's antidepressant activity. Neuropsychopharmacology 33:2303-2312, 2008

25. Yatham LN, Kennedy SH, Schaffer A, et al.: Canadian Network for Mood and Anxiety Treatments (CANMAT) and International Society for Bipolar Disorders (ISBD) collaborative update of CANMAT guidelines for the management of patients with bipolar disorder: Update 2009. Bipolar Disorder 11: 225-255, 2009

26. Chamberlain SR, Muller U, Robbins TW, et al.: Neuropharmacological modulation of cognition. Current Opinion in Neurology 19:607-612, 2006

27. Nathan PJ, Sitaram G, Stough C, et al.: Serotonin, noradrenaline and cognitive function: A preliminary investigation of the acute pharmacodynamic effects of a serotonin versus a serotonin and noradrenaline reuptake inhibitor. Behavioural Pharmacology 11:639-642, 2000

28. Holzer L, Preuss U, Baumgartner L, et al.: Quetiapine in adolescents with non-affective psychotic disorders: An open-label trial. Pharmacopsychiatry 44:87-95, 2011

29. Kay SR, Fiszbein A, Opler LA: The positive and negative syndrome scale (PANSS) for schizophrenia. Schizophrenia Bulletin 13:261-276, 1987

30. Guy W: ECDEU Assessment Manual. Rockville, MD, US Department of Health, Education and Welfare, 1976

31. Reitan R, Wolfson D: The Halstead-Reitan Neuropsychological Test Battery: Theory and Clinical Interpretation. Tucson, AZ, Neuropsychology Press, 1985

32. Asarnow RF, Nuechterlein KH, Subotnik KL, et al.: Neurocognitive impairments in nonpsychotic parents of children with schizophrenia and attention-deficit/hyperactivity disorder: The University of California, Los Angeles Family Study. Archives of General Psychiatry 59:1053-1060, 2002 
33. Nuechterlein KH: Vigilance in Schizophrenia and Related Disorders. In: Steinhauer SR, Gruzelier JH, Zubin J (Eds) Handbook of Schizophrenia: Neuropsychology, Psychophysiology and Information Processing. New York, Elsevier Science Inc., 1991

34. Hebb DO: Distinctive Features of Learning in the Higher Animal. In: Delafresnaye JE (Ed) Brain Mechanisms and Learning. New York, Oxford University Press, 1961

35. Corsi PM: Human Memory and the Medial Temporal Region of the Brain. Dissertation Abstracts International 34:891B (University Microfilms No. AAI05-77717), 1972

36. Rey A: L'examen clinique en psychologie. Paris, Presses Universitaires de France, 1964

37. Osterrieth, P: Le test de copie d'une figure complexe: Contribution à l'étude de la perception et de la mémoire. Archives de Psychologie, Geneva 30:205-220, 1944

38. Spreen O, Strauss E: A Compendium of Neuropsychological Tests: Administration, Norms, and Commentary, 2nd edn. New York, Oxford University Press, 1998

39. Bolognini M, Plancherel B, Laget J, et al.: Evaluation of the adolescent drug abuse diagnosis instrument in a Swiss sample of drug abusers. Addiction 96:1477-1484, 2001

40. Buchanan RW, Davis M, Goff D, et al.: A summary of the FDA-NIMH-MATRICS workshop on clinical trial design for neurocognitive drugs for schizophrenia. Schizophrenia Bulletin 31:5-19, 2005

41. Gothelf D, Apter A, Reidman J, et al.: Olanzapine, risperidone and haloperidol in the treatment of adolescent patients with schizophrenia. Journal of Neural Transmission 110:545-560, 2003

42. Miyake A, Friedman NP, Emerson MJ, et al.: The unity and diversity of executive functions and their contributions to complex "Frontal Lobe" tasks: A latent variable analysis. Cognitive Psychology 41:49-100, 2000

43. Good KP, Kiss I, Buiteman C, et al.: Improvement in cognitive functioning in patients with first-episode psychosis during treatment with quetiapine: An interim analysis. British Journal of Psychiatry Supplement 43:s45-s49, 2002

44. Kivircik Akdede BB, Alptekin K, Kitis A, et al.: Effects of quetiapine on cognitive functions in schizophrenia. Progress in Neuro-Psychopharmacology \& Biological Psychiatry 29:233-238, 2005

45. Arvanitis LA, Miller BG: Multiple fixed doses of "Seroquel" (quetiapine) in patients with acute exacerbation of schizophrenia: A comparison with haloperidol and placebo. The Seroquel Trial 13 Study Group. Biological Psychiatry 42:233-246, 1997

46. Small JG, Hirsch SR, Arvanitis LA, et al.: Quetiapine in patients with schizophrenia. A high- and lowdose double-blind comparison with placebo. Seroquel Study Group. Archives of General Psychiatry 54:549-557, 1997

47. Kahn RS, Fleischhacker WW, Boter H, et al.: Effectiveness of antipsychotic drugs in first-episode schizophrenia and schizophreniform disorder: An open randomised clinical trial. Lancet 371:1085-1097, 2008

48. Robinson DG, Woerner MG, Alvir JM, et al.: Predictors of treatment response from a first episode of schizophrenia or schizoaffective disorder. American Journal of Psychiatry 156:544-549, 1999

49. Robinson DG, Woerner MG, Delman HM, et al.: Pharmacological treatments for first-episode schizophrenia. Schizophrenia Bulletin 31:705-722, 2005

50. Suhara T, Okubo Y, Yasuno F, et al.: Decreased dopamine D2 receptor binding in the anterior cingulate cortex in schizophrenia. Archives of General Psychiatry 59:25-30, 2002

51. Riedel M, Muller N, Strassnig M, et al.: Quetiapine in the treatment of schizophrenia and related disorders. Neuropsychiatric Disease and Treatment 3:219-235, 2007

52. Kapur S, Zipursky R, Jones C, et al.: Relationship between dopamine D(2) occupancy, clinical response, and side effects: A double-blind PET study of first-episode schizophrenia. American Journal of Psychiatry 157:514-520, 2000

53. Kapur S, Zipursky R, Jones C, et al.: A positron emission tomography study of quetiapine in schizophrenia: A preliminary finding of an antipsychotic effect with only transiently high dopamine D2 receptor occupancy. Archives of General Psychiatry 57:553-559, 2000

54. Tandon R, Milner K, Jibson MD: Antipsychotics from theory to practice: Integrating clinical and basic data. Journal of Clinical Psychiatry 60(Suppl 8):21-28, 1999

55. Nikisch G, Baumann P, Wiedemann G, et al.: Quetiapine and norquetiapine in plasma and cerebrospinal fluid of schizophrenic patients treated with quetiapine: Correlations to clinical outcome and HVA, 5-HIAA, and MHPG in CSF. Journal of Clinical Psychopharmacology 30:496-503, 2010

56. Holzer L, Halfon O: Normalization of impaired cognitive functions failed to improve clinical symptomatology in a schizophrenic patient. European Psychiatry 19:384-386, 2004

57. Conway Greig T, Nicholls SS, Wexler BE, et al.: Test-retest stability of neuropsychological testing and individual differences in variability in schizophrenia outpatients. Psychiatry Research 129:241-247, 2004 


\section{Author Biographies}

Sébastien Urben, PhD is a research psychologist in the Unit Research of the University Service of Child and Adolescent Psychiatry, Centre Hospitalier Universitaire Vaudois (CHUV), Lausanne, Switzerland.

Pierre Baumann, MD, Prof is a biochemist/clinical psychopharmacologist, Department of Psychiatry, Centre Hospitalier Universitaire Vaudois (CHUV), Lausanne, Switzerland.

Sandra Barcellona, MSc is an assistant psychologist in the Day Care Unit for Adolescents of the University Service of Child and Adolescent Psychiatry, Centre Hospitalier Universitaire Vaudois (CHUV), Lausanne, Switzerland.

Muriel Hafil, MSc is an assistant psychologist in the Day Care Unit for Adolescents of the University Service of Child and Adolescent Psychiatry, Centre Hospitalier Universitaire Vaudois (CHUV), Lausanne, Switzerland.

Ulrich Preuss, MD, PhD was a senior psychiatrist and a psychologist in the Kinder und Jugendpsychiatrische Poliklinik, Bern, Switzerland. Nowaday, he works in the Klinik für Kinder- und Jugendpsychiatrie, Psychosomatik und Psychotherapie, Brandenburg an der Havel, Germany.

Claire Peter-Favre, $\mathbf{P h D}$ is an assistant psychologist in the University Service of Neuropsychology and Neurorehabilitation, Centre Hospitalier Universitaire Vaudois (CHUV), Lausanne, Switzerland.

Stéphanie Clarke, MD, Prof is a senior psychiatrist in the head of the University Service of Neuropsychology and Neurorehabilitation, Centre Hospitalier Universitaire Vaudois (CHUV), Lausanne, Switzerland.

Olivier Halfon, MD, Prof is a senior psychiatrist in the head of the University Service of Child and Adolescent Psychiatry, Centre Hospitalier Universitaire Vaudois (CHUV), Lausanne, Switzerland.

Laurent Holzer, MD, PD is a senior psychiatrist in the head of the Day Care Unit for Adolescents of the University Service of Child and Adolescent Psychiatry, Centre Hospitalier Universitaire Vaudois (CHUV), Lausanne, Switzerland. 